\title{
Posiciones y debates en torno a los mecanismos de solución de controversias inversor - Estado ${ }^{12}$
}

Positions and debates on investor dispute resolution mechanisms - State

\section{Magdalena Bas Vilizzio}

magdalenabas@gmail.com

Profesora Adscripta de Derecho Internacional Público y Asistente de Comercio Exterior de la Facultad de Derecho de la Universidad de la República (Uruguay).

Resumen: Los mecanismos de solución de controversias inversor - Estado previstos en más de 3000 tratados bilaterales de inversión y otros instrumentos jurídicos han sido centro de numerosas críticas en los últimos años. En esta línea, en primer lugar, se analizarán los riesgos que entraña el sistema arbitral del Centro de Arreglo de Diferencias relativas a Inversiones (CIADI) o la Corte Permanente de Arbitraje (CPA), entre otros, y que han sido identificados desde el ámbito académico, social y gubernamental. Este trabajo analizará específicamente la conformación de los tribunales arbitrales, la ausencia de una instancia de apelación, la inclusión de una amplia diversidad de temas dentro del concepto de inversión protegida y el factor social en el arbitraje de inversiones, especialmente en relación al género y nacionalidad de los árbitros.

Asimismo, se examinarán los diferentes caminos hacia la reforma o intentos de mejoramiento parcial del sistema en base a ejemplos concretos. Tal es el caso de la eliminación de ciertas áreas de la solución de controversias inversor - Estado, como las políticas de control del tabaco del Acuerdo de Asociación Transpacífico; la creación de un sistema de Cortes de Inversiones como propone la Unión Europea en los acuerdos que está negociando con terceros Estados; o la exclusión de los mecanismos de solución de controversias de los acuerdos de protección de inversiones, como en el nuevo modelo de Acuerdo de cooperación y facilitación de inversiones de Brasil.

Palabras clave: Solución de controversias; inversiones; Estado; CIADI

Abstract: Investor - State dispute settlement mechanisms, that are part of more than 3000 bilateral investment treaties and other legal instruments, have been the center of numerous critics during the last years. Therefore, firstly this paper analyses the risks of International Center for Settlement of Investment Disputes (ICSID) or Permanent Court of Arbitration, among others, which were identified by referents in academia, civil society and governments. The paper specifically the designation of arbitrators, the lack of an instance of appeal, the wide concept of protected investment and social factor in investment arbitration, specially arbitrator's gender and nationality.

Artículo enviado el 04.12.2016 y aceptado el 10.01.2017.

Este trabajo es una versión corregida y actualizada de la ponencia presentada en el VIII Congreso de Relaciones Internacionales del Instituto de Relaciones Internacionales (IRI) de la Universidad Nacional de la Plata, La Plata 23, 24 y 25 de noviembre del 2016. Eje temático: Relaciones Económicas Internacionales. 
Additionally, the paper studies some of the different ways towards the investor - State dispute settlement reform or attempts of partial improvement of the system and develops specific examples. For example the carved - out of some areas of investor - State dispute settlement, such as the case of measures of tobacco control of the Transpacific Partnership Agreement; the constitution of an Investment Court System as it is proposed by the European Union in the international treaties that area being under negotiation with third parties; or the exclusion of investor - State dispute settlement of investment protection agreements, for example the new Brazilian model of Cooperation and Facilitation Investment Agreement.

Key words: Dispute settlement; investment, State; ICSID

\section{Introducción}

Más de tres mil instrumentos jurídicos internacionales, entre tratados bilaterales de inversión (TBI), capítulos de inversiones en tratados de libre comercio y otros acuerdos regionales, prevén mecanismos de solución de controversias inversor - Estado, especialmente arbitraje ad hoc en el marco de instituciones como el Centro Internacional de Arreglo de Diferencias relativas a Inversiones (CIADI) del Grupo Banco Mundial, la Corte Permanente de Arbitraje (CPA), la Cámara de Comercio Internacional, entre otras, o siguiendo reglas de procedimiento de la Comisión de Naciones Unidas para el Derecho Mercantil Internacional (UNCITRAL por su sigla en inglés) o creadas para el caso concreto.

No obstante, en los últimos años diversas voces desde la academia, la sociedad civil organizada y los Gobiernos abrieron el debate respecto a los riesgos e inconsistencias en el sistema de solución de controversias inversor - Estado, particularmente del arbitraje ad hoc en el CIADI. En este sentido, en el apartado 2 de este trabajo se analizarán algunas de las críticas, a saber: la inclusión de una amplia diversidad de temas dentro del concepto de inversión protegida por los acuerdos, la ausencia de una instancia de apelación de los laudos arbitrales emitidos por tribunales ad hoc del CIADI, y el desbalance de género y nacionalidades en la conformación de dichos tribunales arbitrales.

Frente a esta situación, a las críticas anteriormente mencionadas y otras, los Estados han tomado diferentes caminos que van desde permanecer en el sistema sin plantear cambios hasta convertirse en disidentes, como es el caso de Bolivia, Ecuador y Venezuela que denunciaron en 2007, 2009 y 2012 el Convenio de Washington constitutivo del CIADI, y en el caso de los dos primeros comenzaron procesos de terminación de los TBI en vigor, habiendo logrado diferente grado de avance ${ }^{3}$.

3 Magdalena Bas. "Algunas reflexiones en torno al retiro de Bolivia, Ecuador y Venezuela del CIADI". Revista Densidades, No. 17, $51-67$

https://www.academia.edu/12981235/Algunas_reflexiones_en_torno_al_retiro_de_Bolivia_Ecuador_y_Venezuela_del_CIADI_ 2015_. [consulta: 24.may.2017]. 
Sin embargo, otros caminos intermedios han cobrado mayor relevancia en los últimos años. En este sentido pueden citarse el Acuerdo de Asociación Transpacífico que exceptúa de las demandas de los inversores a las políticas de control del tabaco; la propuesta de la Comisión Europea de creación de una Corte de Inversiones en el marco de los acuerdos que está negociando la Unión Europea con terceros Estados desde el año 2009; o la exclusión de los mecanismos de solución de controversias inversor - Estado de los acuerdos de protección de inversiones, como en el nuevo modelo de Acuerdo de cooperación y facilitación de inversiones de Brasil. Las diferentes propuestas de reforma, mejoramiento o exclusión del sistema serán el centro de análisis del punto 3 de este trabajo.

\section{Debates en torno al sistema de solución de controversias inversor - Estado: críticas e inconsistencias}

\subsection{Definición amplia de inversión en los acuerdos de protección de inversiones}

El primer punto a analizar refiere a la propia definición de inversión puesto que es en base a ella que se determina si se trata o no de una inversión protegida por el acuerdo en cuestión, y por tanto un incumplimiento de tal protección puede ser pasible de reclamo en vía arbitral. La jurisdicción de los foros internacionales de arbitraje de inversiones suele estar supeditada a una serie de requisitos.

En el caso particular del CIADI, el artículo 25 del Convenio de Washington establece que la institución tiene jurisdicción para entender en aquellas controversias inversor - Estado que sean de naturaliza jurídica, que tengan una relación directa con una inversión entre un Estado parte y un nacional de otro Estado parte de dicho tratado, y en las que exista una cláusula de prórroga de jurisdicción por escrito. Si bien el texto del convenio es claro, no prevé una definición de inversión, por tanto, en caso de excepciones preliminares sobre la jurisdicción, suele recurrirse a la definición prevista en los instrumentos jurídicos en los cuales se ha otorgado la prórroga de jurisdicción, como ser TBI, capítulos de inversiones en tratados de libre comercio, leyes de inversiones, contratos inversor - Estado, otros acuerdos regionales.

Si bien la jurisdicción del CIADI puede quedar restringida a ciertas materias, si así se prevé en el instrumento jurídico en que los Estados otorguen el consentimiento ${ }^{4}$, las definiciones previstas en los TBI y otros instrumentos internacionales suelen ser laxas y dejan dentro una gran multiplicidad de áreas, incluso aquellas vinculadas a políticas públicas soberanas. En este punto es importante traer a colación la controversia entre Philip Morris Brand Sàrl, Philip Morris Products S.A. y Abal Hermanos S.A. y Uruguay (CIADI caso número ARB/10/7), cuyo laudo fue recientemente emitido ${ }^{5}$. El objeto

4 María Fernanda Vásquez. "Arbitraje ante el CIADI: aspectos relevantes y reflexiones sobre su operatividad”. Revista Derecho de la Empresa Legis. Nro. 8.

http://www.arbitrajecomercial.com/BancoConocimiento/A/arbitraje_ante_el_ciadi/arbitraje_ante_el_ciadi.asp. citado el 1 de junio de 2015

5 Un análisis particular del laudo y sus implicancias puede encontrarse en Magdalena Bas Vilizzio. “Gracias por no fumar”. Columna de Opinión, Nueva Sociedad. http://nuso.org/articulo/gracias-por-no-fumar/ [consulta: 24.may.2017]; Martin Dietrich. "Philip 
de la disputa se centra en las medidas tomadas por el Estado relativas a la presentación única por marca comercial y el uso de pictogramas con advertencias sanitarias del 80\% del frente y el reverso de las cajillas en cumplimiento de las disposiciones del Tratado Marco de la Organización Mundial de la Salud sobre Control del Tabaco, del cual Uruguay es parte. Tales disposiciones, a entender de la empresa, representaban un incumplimiento del TBI Uruguay - Suiza en relación al trato justo y equitativo, constituyendo una expropiación sin debida compensación de sus marcas comerciales, derecho incluido dentro de la definición de inversión del acuerdo (artículo 1 numeral 2 literal d).

El 8 de julio de 2016, después de seis años de litigio, el tribunal arbitral ad hoc emite un laudo ${ }^{6}$ que no hace lugar a las pretensiones de la tabacalera, reconociendo el poder soberano de policía del Estado para regular la salud pública y que, por tanto, el Estado no incurre en responsabilidad internacional por las medidas tomadas en el marco de la política antitabaco. En consecuencia, condena a la empresa al pago de los gastos del tribunal arbitral y administrativo del CIADI, así como un reintegro de siete de los poco más de diez millones de dólares de los costos pagados por Uruguay ${ }^{7}$.

Más allá de las particularidades del caso Philip Morris contra Uruguay, es importante tener en cuenta que una definición amplia de inversiones habilita a tribunales de inversión a resolver controversias en las que se debaten políticas públicas relacionadas a la protección de la salud, derecho humano consagrado en diferentes tratados internacionales, como ser la Convención Internacional sobre derechos económicos, sociales y culturales de 1966 (artículo 12), la Convención sobre los derechos del niño de 1989 (artículo 24) y el Protocolo Adicional a la Convención Americana de derechos humanos en materia de derechos económicos, sociales y culturales "Protocolo de San Salvador" de 1988 (artículo 10).

En el punto 3.1 de este trabajo se desarrollarán las características fundamentales del caso Philip Morris Asia contra Australia (CPA caso número 2012 - 2), cuyo inicio también tiene como marco medidas de control del tabaco, y en conjunto con el caso Philip Morris contra Uruguay fueron tomados en consideración durante la negociación del Acuerdo de Asociación Transpacífico.

Morris vs. Uruguay: todas las demandas desestimadas; Uruguay recibirá reembolso por US\$7 millones”. Investment treaty news, No. 3, Vol. 7, 13 - 14. http://www.iisd.org/sites/default/files/publications/iisd-itn-agosto-2016-espanol.pdf [consulta: 24.may.2017]; Felipe Michelini. "Philip Morris versus Uruguay: efectos colaterales". La Diaria, Columna de opinión.

http://ladiaria.com.uy/articulo/2016/8/philip-morris-versus-uruguay-efectos-colaterales/_[consulta: 24.may.2017]; Tania Voon y Andrew Mitchell. "Philip Morris vs. tobacco control: two wins for public health, but uncertainty remains". Columbia FDI

Perspectives, No. 182. http:/ / ccsi.columbia.edu/files/2013/10/No-182-Voon-and-Mitchell-FINAL.pdf [consulta: 24.may.2017].

6 CIADI. Caso número ARB/10/7: Philip Morris Brand Sàr, Philip Morris Products S.A. y Abal Hermanos S. A. contra Uruguay. Laudo del 8 de julio de 2016. https://medios.presidencia.gub.uy/tav_portal/2016/noticias/NO_U130/laudo_spa1.pdf [consulta: 24.may.2017].

7 Si bien el tribunal entiende que sería de aplicación la máxima "el perdedor paga”, dado que las tres excepciones preliminares presentadas por Uruguay fueron rechazadas por el tribunal, no corresponde el pago de la totalidad de los costos incurridos por el Estado.

Número de página no utilizable para citar 


\subsection{CIADI: procedimiento arbitral y ausencia de apelación}

Definiciones amplias de inversión abren la puerta al cuestionamiento de políticas públicas soberanas, no obstante, el problema no se cierra con definiciones restrictivas puesto el propio procedimiento arbitral en el CIADI tiene carencias, en particular la falta de apelación. En este sentido, dicho procedimiento arbitral puede ser dividido en cinco etapas. La primera de ellas es la presentación de la solicitud de iniciar un arbitraje y su registro por parte del Secretario General (artículo 36 del Convenio de Washington). A posteriori, se constituye el tribunal ad hoc, compuesto por un único árbitro o un número impar de árbitros designados, de acuerdo a lo previsto por las partes (artículo 37).

La tercera etapa del proceso refiere a las actuaciones escritas, esto es, la presentación de un memorial por parte del actor y el correlativo memorial de contestación del demandado, ambos conteniendo una relación de hechos, normas aplicables y las peticiones. Eventualmente, estas presentaciones son seguidas por una réplica y una dúplica de actor y demandado, respectivamente.

Asimismo, desde la reforma de las Reglas procesales aplicables a los procedimientos de arbitraje del año 2006, se prevé la posibilidad de presentación de terceros, ya sea persona física o jurídica, en calidad de amicus curiae mediante un documento escrito en relación a las cuestiones de fondo del asunto (Regla 37 (2)). La intervención de amicus curiae o amigos de la corte tiene como fin la colaboración al tribunal en el proceso de toma de decisiones de un tercero con un interés significativo en el caso y que posee conocimiento o visión particular, diferente a la perspectiva de las partes en la controversia. Su participación debe ser consultada a las partes, y el tribunal debe asegurarse que esta no interfiera en el procedimiento, genere una carga indebida o perjudique injustamente a una de las partes ${ }^{8}$. En el caso Philip Morris contra Uruguay, por ejemplo, fue aceptada la presentación como amicus curiae de la Organización Mundial de la Salud y la Secretaría del Convenio Marco sobre Control del Tabaco.

La siguiente etapa corresponde a las actuaciones orales, conformadas por las audiencias destinadas a que el tribunal escuche a las partes, sus representantes legales, testigos y peritos. En base a los hechos y el Derecho aplicable al caso, el tribunal dicta un laudo por mayoría de votos, el cual debe ser motivado, y no será publicado sin el consentimiento de las partes ${ }^{9}$ (artículo 48). Dicho laudo es definitivo, y si es pasible de recurso de aclaración, revisión y anulación (artículo 50 a 52).

Ni el recurso de revisión ni el de anulación tienen las características de una auténtica apelación. El primer caso, se basa en el descubrimiento de hechos que hubieran influido decisivamente en el laudo. El recurso de anulación, por otra parte, puede fundarse en causas gravísimas de procedimiento o forma en la emisión del laudo. El artículo 52 las enumera taxativamente: 1) constitución incorrecta del tribunal; 2) actuación del tribunal extralimitándose manifiestamente en sus facultades; 3) corrupción de un miembro del tribunal; 4) violación grave de unas normas de procedimiento; 5) emisión de un

Eugenia Levine. "Amicus Curiae in International Investment Arbitration: The Implications of an Increase in Third-Party Participation”. Berkeley Journal of International Law, Vol. 29, Nro. 1, 200 - 224. http:/ /dx.doi.org/doi:10.15779/Z386D49 [consulta: 24.may.2017].

9 Previa o conjuntamente con la decisión sobre el fondo del asunto, el tribunal puede resolver las cuestiones relativas a su competencia, en caso de ser solicitado.

Número de página no utilizable para citar 
laudo no motivado. A octubre de 2016, únicamente en 90 de los 586 arbitrajes administrados por el CIADI se presentó recurso de anulación, conforme a datos del sitio web del CIADI.

\subsection{El factor social en la conformación de los tribunales arbitrales ad hoc: desbalance de género y nacionalidades}

A las críticas anteriormente examinadas, puede sumarse el desbalance en dos aspectos centrales del capital social en el arbitraje de inversiones, esto es, las características sociales de los árbitros que, hasta el momento, han sido designados para conformar los tribunales ad hoc: la nacionalidad y el género. Las últimas estadísticas disponibles publicadas por el CIADI ${ }^{10}$ muestran que de las 103 nacionalidades de los árbitros, conciliadores y miembros de comités ad hoc designados bajo el Convenio de Washington y el Mecanismo Complementario, poco más de los dos tercios corresponden a Europa Occidental y América del Norte sumadas. De hecho, la mitad de las designaciones se obtiene sumando siete nacionalidades, a saber: Francia, Estados Unidos, Reino Unido, Canadá, Suiza, España y Austria ${ }^{11}$.

En cuanto a otro factor esencial del capital social, el género, las cifras son contundentes. Los resultados de la investigación realizada por Puig muestran que, a 2014, el 93\% de las designaciones de árbitros para tribunales ad hoc del CIADI corresponde a hombres, más aun, dos mujeres dominan las designaciones dentro del 7\% correspondiente al género femenino ${ }^{12}$. Ampliando la mirada a otros foros arbitrales los números se mantienen casi no cambiados. En este sentido, Greenwood y Baker muestran que los tres foros por ellos analizados, el CIADI, la Cámara de Comercio de Estocolmo y la Corte de Arbitraje Internacional de Londres, únicamente el 6\% de las designaciones de árbitros corresponden a mujeres ${ }^{13}$. Estas cifras manifiestan un claro desbalance de género, que sigue la lógica del desbalance de nacionalidades.

A este punto cabe destacar que los propios Estados son parcialmente responsables del desbalance anteriormente indicado, puesto que en el caso específico del CIADI, cada una de las partes designa un árbitro y el tercero es mutuamente acordado, o en su defecto, designado por el propio Centro, conforme al artículo del Convenio de Washington.

10 CIADI. Carga de casos del CIADI - Estadísticas (Edición 2016 - 2).

https://icsid.worldbank.org/apps/ICSIDWEB/resources/Documents/ICSID\%20Web\%20Stats\%202016-

2\%20(Spanish)\%20Final.pdf [consulta: 24.may.2017].

11 Estas cifras no tienen en cuenta las dobles nacionalidades, ni el país de formación universitaria de los árbitros, conciliadores y miembros de comités ad hoc, de ser así los porcentajes serían aún mayores a favor de los países centrales, confirmando la tendencia.

12 Sergio Puig. "Social Capital in the Arbitration Market". The European Journal of International Law, Vol. 25, No. 2, 387 - 424. http://www.ejil.org/pdfs/25/2/2495.pdf [consulta: 24.may.2017].

13 Lucy Greenwood y C. Mark Baker. "Getting a better balance on international arbitration tribunals". Arbitration International. The Journal of the London Court of International Arbitration, Vol. 28, No. 4, 653 - 667.

http://www.cpradr.org/Portals/0/Resources/Articles/ARBI\%2028-4_Lucy\%20Greenwood_Offprint.pdf [consulta: 24.may.2017].

Número de página no utilizable para citar 


\section{Posiciones en torno a la reforma, mejoramiento o exclusión del sistema}

\subsection{Excepciones sectoriales a los mecanismos de solución de controversias inversor - Estado: el Acuerdo de Asociación Transpacífico ${ }^{14}$}

Una primera posición respecto al mejoramiento del sistema refiere a la exclusión de ciertos sectores del arbitraje inversor - Estado, especialmente aquellos en los que se busca implementar políticas públicas para proteger bienes jurídicos que se consideran superiores. Este es el caso de uno de los mega acuerdos actualmente en proceso de ratificación, el Acuerdo de Asociación Transpacífico (Acuerdo TPP por su sigla en inglés), cuyo tratado fundacional actualmente se encuentra en proceso de ratificación. Este tratado se caracteriza por las múltiples excepciones en todos los capítulos, incluso su preámbulo, ya sean excepciones a principios generales, excepciones a excepciones, exclusiones explícitas o implícitas, aclaraciones, limitación de la normativa aplicable, etc cuyo análisis excede el objetivo de este trabajo. ${ }^{15}$.Puntualmente, en el caso específico de la solución de controversias inversorEstado, existen excepciones de diversa índole, pero se destaca la innovación relativa a las medidas de control del tabaco (artículo 29.5), tal vez una de las pocas innovaciones introducidas por el acuerdo.

La norma indica que un Estado parte puede optar por que las medidas de control de tabaco que decida tomar no sean susceptibles de reclamaciones en el marco de la Sección B del Capítulo 9, y por tanto un inversor nacional de otro Estado parte no pueda iniciar un arbitraje por tales motivos. La opción puede ser ejercida en cualquier momento, incluso al inicio o durante el proceso arbitral.

La inclusión de esta disposición responde a las características especiales del tabaco, como único bien de consumo que cuenta con un tratado internacional que controla y previene su consumo ${ }^{16}$, el Convenio Marco de la Organización Mundial de la Salud para el Control del Tabaco, celebrado el 21 de mayo de 2003 y en vigor desde el 27 de febrero de 2005, que cuenta con 180 Estados parte ${ }^{17}$. Asimismo, dos controversias de inversiones relativas al tema fueron tomadas en cuenta por los redactores del acuerdo: el caso Philip Morris contra Uruguay descripto en el punto 2.1 y el caso Philip Morris contra Australia en la CPA (caso número 2012 - 2) ${ }^{18}$.

14 Este apartado sigue parte del abordaje del siguiente artículo: Magdalena Bas Vilizzio. "Solución de controversias inversor - Estado en los nuevos tratados de integración regional: el caso del Acuerdo de Asociación Transpacífico". Revista de la Secretaría del Tribunal Permanente de Revisión, No. 8, 2016, 246 - 264 . http://www.revistastpr.com/index.php/rstpr/article/view/206 [consulta: 24.may.2017].

15 Thomas Bollyki. "TPP tobacco exception proves the new rule in trade". Council for Foreign Relations. http://www.cfr.org/trade/tpp-tobacco-exception-proves-new-rule-trade/p37509 [consulta: 24.may.2017].

16 Thomas Bollyki. "TPP tobacco exception proves the new rule in trade". Council for Roreign Relations.. http://www.cfr.org/trade/tpp-tobacco-exception-proves-new-rule-trade/p37509 [consulta: 24.may.2017].

17 Información disponible en el sitio web de la Organización Mundial de la Salud: http://www.who.int/fctc/signatories_parties/es/ [consulta: 24.may.2017].

18 CPA. Caso 2012 - 2: Philip Morris Asia Limited contra la Mancomunidad de Australia. Laudo de jurisdicción y admisibilidad del 17 de diciembre de 2015. https://pcacases.com/web/sendAttach/1711 [consulta: 24.may.2017].

Número de página no utilizable para citar 
En este sentido, Australia y Nueva Zelanda fueron dos de los Estados parte del Acuerdo TPP que más presionaron para que la mencionada norma fuera una realidad ${ }^{19}$. Como se mencionará, el primer Estado tiene la experiencia de la demanda de Philip en base a la Ley de empaquetado genérico de los cigarrillos (2011) y sus normas modificatorias y complementarias. El argumento del inversor se basaba en la supuesta violación de las disposiciones del TBI Hong Kong - Australia relativas al trato justo y equitativo y expropiación de la propiedad intelectual. El 17 de diciembre de 2015, el tribunal aceptó la objeción de Australia en relación a que constituye abuso de derecho que el demandante realizara una restructuración en Hong Kong, poco antes del inicio del arbitraje.

El caso de Nueva Zelanda, diferente al anterior, se centra en que, en vistas de la demanda recibida por su vecino, el Gobierno decide suspender la sanción del proyecto de ley sobre empaque genérico de cigarrillos que había sido ingresado al Parlamento en el año 2013. Un mes después de concluido el arbitraje contra Australia, el Primer Ministro neozelandés, John Key, anuncia públicamente que la ley de empaque genérico será sancionada en el corto plazo $^{20}$. Este hecho confirma las ideas propuestas por el Experto Independiente sobre la promoción de un orden internacional democrático y equitativo de Naciones Unidas, Alfred - Maurice de Zayas ${ }^{21}$, al sostener que las demandas o amenazas de demandas en áreas de interés público pueden derivar en una parálisis o congelación normativa, o como indica Arato, convertir a las empresas en creadoras de Derecho ${ }^{22}$.

En suma, siguiendo a Johnson y Sachs ${ }^{23}$ puede señalarse que la excepción de las medidas de control del tabaco es necesaria para proteger a los Estados de reclamos de las tabacaleras, como los casos de Uruguay y Australia, pero que el acuerdo pierde la oportunidad de pronunciarse sobre otras áreas objeto de políticas públicas soberanas, como ser el medio ambiente, la salud en general o la seguridad pública.

19 Independientemente del impulso de Australia y Nueva Zelanda en este punto, son interesantes los cambios que este tratado implica a la posición de ambos frente a la solución de controversias inversor- Estado. Según Álvarez, ambos países optaron por excluirla de los TBI celebrados desde el año 2011 (año en que se oficializa la demanda de Philip Morris Asia). En este sentido ver José Álvarez (2016). “Is the Trans-Pacific Partnership's investment chapter the new “gold standard”?” Working Paper IILJ 2016/3 MegaRed Series. https://wp.nyu.edu/megareg/wp-content/uploads/sites/3134/2016/03/Alvarez_IILJ-MegaReg_2016-3.pdf [consulta: 24.may.2017].

20 Kate McHaffie. "New Zealand to implement plain packaging for tobacco". Lexology. http://www.lexology.com/library/detail.aspx?g=2f267230-efb7-4348-bb47-ee53c89bd601 [consulta: 24.may.2017].

21 ONU. Cuarto reporte del Experto Independiente sobre la promoción de un orden internacional democrático y equitativo. Documento A/70/285. http://www.un.org/en/ga/search/view_doc.asp?symbol=A/70/285\&referer=http://www.ohchr.org/EN/Issues/IntOrder/Pag es/IEInternationalorderIndex.aspx\&Lang=S [consulta: 24.may.2017].

22 Julian Arato. "Corporations as Lawmakers." Harvard International Law Journal. Summer 2015. Volume 56, No. 2, 229 - 295 (2015, Boston, Harvard University)

23 Lise Johnson y Lisa Sachs. "The TPP's investment chapter: entrenching, rather and reforming, a flawed system". CCSI Policy Paper (2015 [citado el 1 de julio de 2016], Nueva York, Columbia Center on Sustainable Investment, Columbia University). http://ccsi.columbia.edu/files/2015/11/TPP-entrenching-flaws-21-Nov-FINAL.pdf [consulta: 24.may.2017].

Número de página no utilizable para citar 


\subsection{Nuevas estructuras institucionales: el Acuerdo de la Asociación Transatlántica de Comercio e Inversión}

La propuesta presentada por la Comisión Europea en el marco de las negociaciones del Acuerdo de la Asociación Transatlántica de Comercio e Inversión (T'TIP por su sigla en inglés) respecto a la solución de controversias inversor - Estado, constituye una segunda posición que plantea avanzar hacia la construcción de una nueva estructura institucional entre ambas partes del acuerdo, esto es, un sistema de cortes de inversiones. El texto recoge los resultados de la consulta pública en línea relativa a la protección de inversiones y el sistema de solución de controversias inversor - Estado en el mencionado acuerdo, desarrollada entre el 13 de marzo y 14 de julio de $2014^{24}$.

El sistema de cortes de inversiones, previsto en la Sección III del Capítulo II (Inversiones), se compone por dos niveles: un Tribunal de Primera Instancia (artículo 9) compuesto por quince jueces (cinco nacionales de la Unión Europea, cinco de Estados Unidos, y cinco de terceros Estados). Los jueces deben poseer las calificaciones requeridas en el país de su nacionalidad para ser juez o ser juristas de reconocida competencia, y son elegidos por un período de seis años renovable por una única vez. Asimismo, cada caso concreto será resuelto por tres de los quince jueces, manteniéndose las proporciones de nacionalidades previstas para el Tribunal en pleno, y será presidido por el juez nacional del tercer Estado.

Adicionalmente, el texto prevé un Tribunal de Apelaciones permanente (artículo 10), cuya función es oficiar de segunda instancia ante los fallos del Tribunal de Primera Instancia. El hecho que sea un tribunal permanente mitiga parte de las críticas al actual sistema de solución de controversias Inversor - Estado, especialmente el CIADI, dado que los tribunales se conforman para el caso concreto y carece de una instancia de apelación.

Cabe destacar que el Tribunal de Apelaciones está compuesto por seis miembros (dos nacionales de la Unión Europea, dos de Estados Unidos, y dos de terceros Estados), pudiéndose elevar su número en múltiplos de tres. También es exigido que los miembros cuenten con calificaciones para ocupar los más altos tribunales judiciales de su país o sean juristas de reconocida competencia, y son elegidos por un período de seis años renovable por una única vez.

Con el fin de garantizar la transparencia en los procedimientos y la independencia de tanto los jueces del Tribunal de Primera Instancia como los miembros del Tribunal de Apelaciones, estos deberán seguir un estricto Código de Conducta y no podrán actuar como abogados en ningún caso nuevo o pendiente bajo el acuerdo en cuestión o cualquier otro, así como las jurisdicciones domésticas (artículo 10). Este punto es crucial en el sistema, ya que es una de las críticas más fuertes al actual sistema de solución de controversias inversor - Estado, en particular desde la sociedad civil organizada ${ }^{25}$.

24 La metodología aplicada y los resultados de la consulta pública están disponibles en: http://trade.ec.europa.eu/doclib/docs/2015/march/tradoc_153304.pdf [consulta: 24.may.2017].

25 En este sentido véase especialmente: Pia Eberhardt y Cecilia Olivet. Cuando la injusticia es negocio. m Bruselas/Ámsterdam, The Transnational Institute). https://www.tni.org/files/download/cuando_la_injusticia_es_negocio-web.pdf [consulta: 24.may.2017]. 
Esta propuesta de una nueva estructura institucional ha generado algunas críticas en la doctrina. Se destaca, en primer lugar, el análisis de Sornarajah ${ }^{26}$ que considera que el sistema propuesto no resuelve los problemas de los mecanismos actuales, dado que per se no asegura legitimidad, no hay una determinación clara de la distribución geográfica de los jueces o miembros que corresponden a terceros Estados, y si la hubiera, se tratará de una minoría cuya incidencia no pesa en las decisiones finales. Asimismo, en relación a dichas decisiones, autores como Gaffney ${ }^{27}$ consideran que el borrador se queda a mitad de camino entre la construcción de un nuevo sistema y la permanencia en el actual.

$\mathrm{Schill}^{28}$, por su parte, es crítico frente a la relación entre el sistema de cortes de inversiones propuesto y los tribunales domésticos, punto especialmente criticado en el sistema actual. En este sentido, la ausencia de requisito de agotamiento previo de la jurisdicción doméstica puede poner en tela de juicio la autoridad y legitimidad democrática de los tribunales nacionales, así como quitarles la oportunidad de corregir posibles medidas arbitrarias, discriminatorias o ilegítimas adoptadas por el Gobierno, con la consecuente falta de responsabilidad internacional.

Finalmente, el análisis de la nueva institucionalidad debe ser complementado con la propuesta de la Comisión Europea a nivel multilateral. En este sentido, la institución ha planteado su interés de trabajar con otros Estados y en forma paralela en la conformación de un Tribunal internacional de inversiones de carácter multilateral y permanente. Esta idea además de estar reflejada en el comunicado de prensa que introduce el nuevo sistema de cortes de inversión del Acuerdo TTIP ${ }^{29}$, va de la mano con el artículo 12 del mencionado borrador, en el entendido de que dichas normas dejarán de aplicarse de entrar en vigor un instrumento jurídico internacional que prevea un tribunal multilateral de inversiones o regule un mecanismo multilateral de apelación.

Dado que el sistema multilateral tiene como fin sustituir a los actuales mecanismos de solución de controversias inversor - Estado, es posible concluir que el sistema bilateral estará supeditado a un eventual sistema multilateral hacia donde caminan los debates sobre la reforma de los mecanismos de solución de controversias inversor - Estado.

26 Muthucumaraswamy Sornarajah. “An International Investment Court: panacea or purgatory?” Columbia FDI Perspectives, No. 180. Nueva York, Columbia Center on Sustainable Development, Columbia University): disponible en http://ccsi.columbia.edu/files/2013/10/No-180-Sornarajah-FINAL.pdf [consulta: 24.may.2017].

27 John Gaffney. "The EU proposal for an Investment Court System: what lessons can be learned from the Arab Investment Court?” Columbia FDI Perspectives, No. 181. (2016 [citado el 26 de septiembre de 2016], Nueva York, Columbia Center on Sustainable Development): disponible en http://ccsi.columbia.edu/files/2013/10/Perspective-Gaffney-Final-Formatted.pdf

28 Stephan W. Schill. "The European Commission's Proposal of an "Investment Court System" for TTIP: Stepping Stone or Stumbling Block for Multilateralizing International Investment Law?" Insights, Vol. 20, No. 9. (22 de abril de 2016 [citado el 4 de diciembre de 2016], Washington, American Society of International Law (ASIL)): disponible en https://www.asil.org/insights/volume/20/issue/9/european-commissions-proposal-investment-court-system-ttip-stepping

29 El comunicado de prensa de la Comisión Europea de fecha 16 de septiembre de 2015 se encuentra disponible en: http://europa.eu/rapid/press-release_IP-15-5651_es.htm [consulta: 24.may.2017].

Número de página no utilizable para citar 


\subsection{Exclusión de los mecanismos del arbitraje inversor - Estado de los acuerdos de protección de inversiones: los acuerdos de cooperación y facilitación de inversiones de Brasil}

Finalmente, en tercer lugar, puede citarse la posición más extrema, esto es, el nuevo modelo de acuerdo de promoción y protección de inversiones de Brasil, los acuerdos de cooperación y facilitación de inversiones (ACFI), que elimina de su articulado el tradicional arbitraje inversor - Estado. A octubre de 2016, Brasil ha firmado seis ACFI, tres con Estados africanos lusófonos, esto es, Mozambique (30 de marzo de 2015), Angola (1 de abril de 2015) y Malaui (25 de junio de 2015), y cuatro con Estados Latinoamericanos, a saber: México (26 de mayo de 2015), Colombia (9 de octubre de 2015) y Chile (23 de noviembre de 2015), Perú (28 de abril de 2016). Se encuentran en negociación los acuerdos con Argelia, Marruecos, Sudáfrica y Túnez ${ }^{30}$.

Históricamente Brasil fue un externo al sistema de solución de controversias inversor - Estado, puesto que si bien celebra 14 tratados bilaterales de inversión entre 1994 y $1999^{31}$, cuando seis de ellos llegan al Congreso Brasilero ${ }^{32}$, un Grupo de Trabajo Interministerial, creado durante la Presidencia de Cardozo, concluye que es conveniente retirar estos tratados del Congreso por violar normas constitucionales ${ }^{33}$.

No obstante, dado que en la última década el flujo de inversiones en Brasil ha dejado de ser exclusivamente unidireccional para ser bidireccional, la dirección de la Secretaría de Comercio Exterior del Ministerio de Desarrollo, Industria y Comercio elaboró, en el año 2014, un nuevo modelo de tratado de inversiones. Este excluye los mecanismos de solución de controversias inversor - Estado, previendo únicamente un sistema de prevención de controversias Estado - Estado basado en: 1) la existencia de Puntos Focales u Ombudsman (defensores del pueblo) en cada Estado para la prevención de disputas y facilitación de su resolución; 2) la conformación de un Comité Conjunto, compuesto por representantes gubernamentales de ambos Estados, que se encarga de evaluar la controversia mediante consultas y negociaciones entre las partes, como requisito previo para el inicio de la vía arbitral Estado - Estado.

Cada acuerdo presenta particularidades en el arbitraje Estado - Estado a destacar. Los ACFI firmados con Angola, Malaui y Mozambique prevén que si las partes deciden someterse al arbitraje pueden optar por cualquier mecanismo de solución de controversias en materia de inversiones (artículos 15, 13 y 15 respectivamente). El ACFI con México, además de lo anterior, agrega la posibilidad de crear un tribunal arbitral ad hoc (artículo 19). Asimismo, el acuerdo celebrado con Colombia, por su parte,

30 Jonathan Hamilton y Michelle Grando. "O modelo de proteção de investimentos do Brasil: os novos acordos internacionais". Pontes, 2016. Vol. 12, No. 1, 13 - 17. Disponible en http://www.ictsd.org/sites/default/files/review/Pontes_12-1.pdf [consulta: 24.may.2017].

31 TBI celebrados con Alemania; Bélgica y Luxemburgo; Chile; República de Corea; Cuba; Dinamarca; Finlandia; Francia; Italia; Países Pajos; Portugal; Reino Unido; Suiza; Venezuela.

32 En 1996, los TBI con Suiza, Portugal, Chile y Reino Unido; y en 2000, con Francia y Alemania. En este sentido véase: Déborah Bithiah De Azevedo. (2001). Os acordos para a promoção e a proteção recíproca de investimentos assinados pelo Brasil, http://www2.camara.leg.br/documentos-e-pesquisa/publicacoes/estnottec/tema3/pdf/102080.pdf_[consulta: 24.may.2017].

33 Esteban Actis. "Brasil y la promoción de Tratados Bilaterales de Inversión: El fin de la disyuntiva". Latin American Journal of International Affairs, 2014. Vol. 6, No. 1, 18 - 33. Disponible en http://www.lajia.net/volumenes/LAJIA\%20vol6\%20n1\%20Art02.pdf?attredirects=0\&d=1 [consulta: 24.may.2017].

Número de página no utilizable para citar 
refiere al arbitraje mediante un tribunal ad hoc o, en su defecto, cualquier institución arbitral permanente (artículo 23). Asimismo, tiene la particularidad de excluir del arbitraje las siguientes áreas: responsabilidad social corporativa, lucha contra la corrupción y la ilegalidad, medio ambiente, asuntos laborales, salud y seguridad conforme a su regulación en el propio tratado.

En suma, los ACFI resuelven parte de los problemas tradicionalmente que atañen a los TBI, esto es, mitigan los riesgos y eventuales controversias con la presencia del Ombudsman; y eliminan las típicas cláusulas draconianas que brindan mayores ventajas a los inversores extranjeros frente a los nacionales ${ }^{34}$, como la posibilidad unilateral de presentar acciones ante tribunales arbitrales internacionales.

\section{Reflexiones finales}

A lo largo de este trabajo se analizaron los diferentes debates en la academia, la sociedad civil y los gobiernos respecto al tradicional arbitraje inversor - Estado, especialmente en el caso del arbitraje ad hoc en el CIADI o la CPA. Si bien las críticas son diversas, se optó por analizar tres de ellas, la definición amplia de inversión que habilita demandas en áreas de interés público relativas a políticas estatales inherentes a la soberanía, la ausencia de una instancia de apelación a los laudos de los tribunales del CIADI, y el desbalance de nacionalidades y género a la hora de conformar los tribunales arbitrales, elemento sustancial desde el punto de vista del factor social en la solución de controversias.

Las críticas han encontrado respuesta en propuestas puntuales de reforma o mejoramiento del sistema actual. La excepción a las medidas de control del tabaco prevista en el Acuerdo TPP es un paso enorme en la exclusión de otras áreas relacionadas a derechos humanos y políticas públicas de interés para los Estados, no obstante, es un paso corto y reactivo frente a las demandas de la tabacalera Philip Morris a Uruguay y Australia. Cabe preguntarse si no hubiera sido el momento justo para excluir otras áreas, como ser la seguridad nacional, el medio ambiente, la salud pública en general, o ciertos temas en particular (ejemplo: control del alcoholismo, alimentos transgénicos, alimentos con alto contenido de azúcar), acceso al agua potable, o incluso los derechos humanos en general.

Un paso más adelante lo constituye la propuesta de la Comisión Europea que avanza hacia una nueva institucionalidad que resuelve parte de los problemas analizados en este trabajo, incluyendo una instancia de apelación y criterios más equitativos en la distribución de nacionalidades de los árbitros.

34 Esteban Actis. "Brasil frente al orden internacional liberal (2011-2013). Los límites de la posición reformista a la luz del régimen internacional de inversiones”. Mural Internacional, Vol. 6, No. 1, 22 - 34 (2015). Programa de Pós-Graduação em Relações Internacionais da Universidade do Estado do Rio de Janeiro. Disponible en http:/ /www.epublicacoes.uerj.br/index.php/muralinternacional/article/view/12838/13564 [consulta: 24.may.2017].

Número de página no utilizable para citar 
Aun así, la mayoría de los miembros de los tribunales reiterarán el binomio Europa Occidental América del Norte, que no incorpora la visión de género, y no se trata de una institución multilateral.

Finalmente, el caso de Brasil presenta otras connotaciones, que tienen su germen en la propia relación histórica de este país respecto al sistema internacional de protección de inversiones. Sin haber sido parte nunca, ingresa a este, pero prescinde de uno de sus elementos fundamentales: el arbitraje inversor - Estado como mecanismo de solución de controversias. Este es un camino de salida en torno a las críticas y es perfectamente compatible con un sistema multilateral de cortes inversor - Estado.

Sea cual sea el camino, la situación actual evidencia una imperiosa reforma o mejoramiento del sistema de solución de controversias inversor - Estado, pero cualquiera sea el camino que decida transitarse, debe hacer en protección de la soberanía estatal. De lo contrario, las demandas o amenazas de demandas de los inversores continuarán derivando en un congelamiento normativo, hecho que evidencia una disminución del espacio regulatorio inherente a la soberanía estatal y pone en jaque a las políticas públicas de protección de la salud, medioambiente, acceso al agua, entre otros. 